
for Mechanical Engineering

\title{
Description of the algorithms to test the horizontal jump in a computerized testing system of gross motor skills
}

\begin{tabular}{|r|l|}
\hline Journal: & Transactions of the Canadian Society for Mechanical Engineering \\
\hline Manuscript ID & TCSME-2021-0069.R1 \\
\hline Manuscript Type: & Article \\
\hline Author: & 09-Jul-2021 \\
\hline $\begin{array}{r}\text { Complete List of Authors: } \\
\text { CHEBmitted by the }\end{array}$ & $\begin{array}{l}\text { Moraes, Arina; Laurentian University, School of Engineering } \\
\text { Tremblay, Line; Laurentian University, School of Human Kinetics } \\
\text { Thorpe, Moriah; Laurentian University, School of Human Kinetics } \\
\text { Theriault, Francis; Laurentian University, School of Human Kinetics }\end{array}$ \\
\hline $\begin{array}{r}\text { Keywords: } \\
\text { Is the invited manuscript for } \\
\text { consideration in a Special } \\
\text { Issue? : }\end{array}$ & $\begin{array}{l}\text { Gross motor skills, Horizontal jump, Kinect, Test of gross motor } \\
\text { development }\end{array}$ \\
\hline
\end{tabular}

\section{SCHOLARONE \\ Manuscripts}




\section{Description of the algorithms to test the horizontal jump in a computerized testing} 2 system of gross motor skills

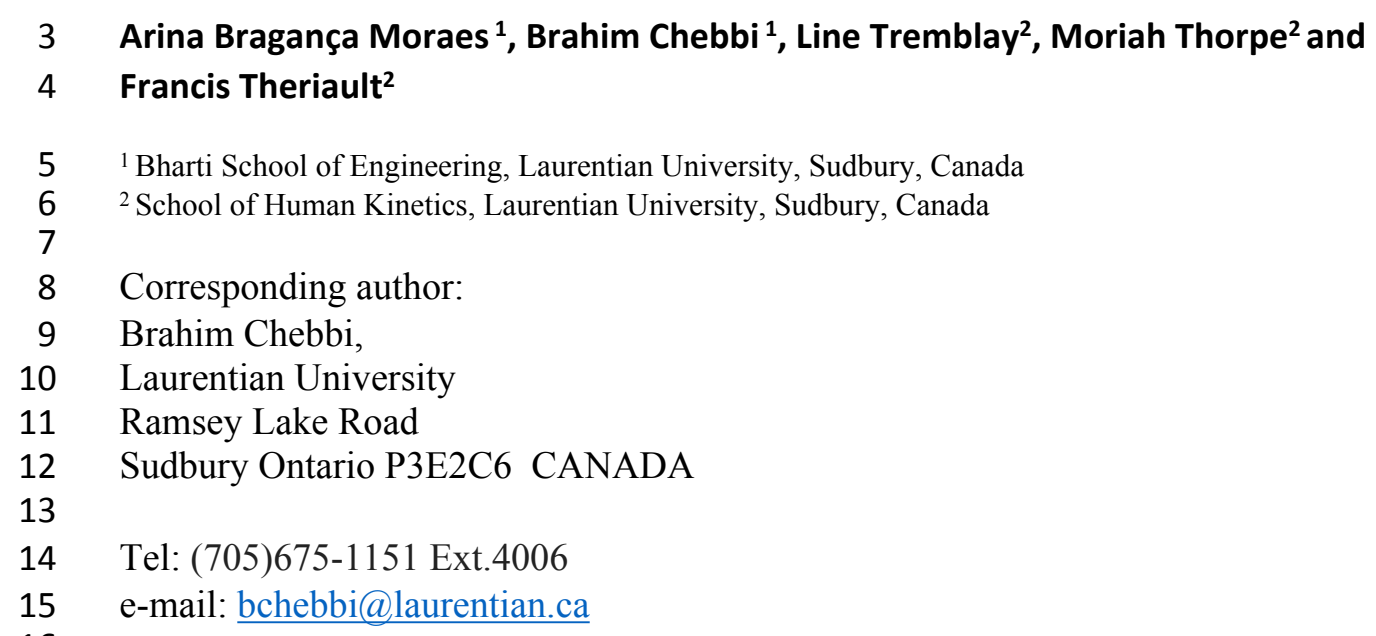




\section{Abstract}

18 Gross motor skills assessment is important for children, youth and adults and can be beneficial in the study and diagnosis of 19 different health conditions. Classical tools are administered mainly by operators. A computerized system using sensors to assess the gross motor skills would enhance these tools, providing consistency of measurements, reduced human error and automated data collection. As part of a computerized system to measure gross motor skills using the Kinect ${ }^{\mathrm{TM}}$, we present the details of development of the algorithms to test the horizontal jump (HJ) skill. These algorithms were developed by translating the performance criteria into equations of the coordinates of anatomical landmarks describing an expected kinematic behaviour of relevant body parts. Testing of the algorithms showed that they were successful in correctly evaluating the performance criteria.

Keywords: Gross motor skills, Horizontal jump, Kinect, Test of gross motor development. 


\section{Introduction}

Children who show developmental delay or lower motor skills than expected tend to experience difficulty when performing physical activities, reducing their enthusiasm for an active lifestyle, which directly impacts their health status and quality of life (Booth et al. 1999). Moreover, evidence suggests that unhealthy habits acquired during childhood such as sedentary behaviours, are likely to persist during adolescence and adulthood (Zecevic et al. 2010). Motor skills assessment permits actual performance measurement and documentation of the changes in a person's motor skill development (VoelckerRehage 2008). Gross motor skills assessment is important for children, youth and adults and can be beneficial in the study and diagnosis of different conditions such as obesity (Frey and Chow 2006), cerebral palsy (McCormick et al. 2007) and Parkinson's disease (Galna et al. 2014). Early assessment is important because it allows identification of problems associated with poor motor competence, such as obesity, learning disabilities, coordination disorders and psychosocial maladjustment (Skinner and Piek 2001). An early diagnosis of poor gross motor skills can help their improvement with the assistance of a qualified professional (Goodway et al. 2003).

Gross motor skills performance can be measured using diverse assessment tools (Griffiths et al. 2018). For example, the Test of Gross Motor Development (TGMD) evaluates 13 gross motor skills (Table 1). The examiner follows instructions on a scoring sheet which specifies the performance criteria for each skill, in order to assess the quality of the movement. Classical tools, such as the TGMD, are administered by operators. Consequently, a limitation of these tools is the total dependence on human observation, which can lead to mistakes and poor judgment of the results (Tremblay et al. 2016; Bonifacci 2004). It is also expected that the results vary according to the examiners skills and experience. In addition, depending on the type of test, the cost of administration and analysis can be very high.

A computerized system using sensors to assess the gross motor skills has the potential to circumvent these limitations. It would have the advantages of consistency of measurements, reduced human error and automated data collection and analysis. The assessment of gross motor skills using sensors was reported by a few researchers. In particular, Inertial Measurements Units (IMUs) were used to assess the TGMD-2 locomotor skills (Bisi et al. 2017), the childhood hopping developmental level (Masci et al. 2012) and the ball overarm throwing (Grimpampi et al. 2016). IMUs are reliable and accurate, however many of them are needed to assess a particular skill, which could make it uncomfortable for the participant to have them attached while performing the skill. Specialized motion capture devices such as the Vicon ${ }^{\mathrm{TM}}$ are used for fitness and health applications. These devices are very accurate, but usually expensive and rely on markers positioned on different parts of the subject body, which requires significant setup time. 
Many marker-less motion capture systems were developed for the gaming industry and are available at an affordable

cost because of the large customer base for this industry. For example, the launch of the Microsoft Kinect ${ }^{\mathrm{TM}}$ marked a revolutionary advancement for gaming developers, thanks to its affordable price and depth camera. It tracks body movements and determines the position of anatomical landmarks in 3D space. This makes it a potentially useful tool to record and evaluate gross motor skills (Gray et al. 2017; Tremblay et al. 2016). Kinect ${ }^{\mathrm{TM}}$ for Windows was discontinued in 2017 and a new system, Azure Kinect ${ }^{\mathrm{TM}}$, was launched in 2019. Many other systems are available in the market and offer similar functionality and Software Development Kit (SDK) support.

The present work is part of a larger investigation (Tremblay et al. 2016) which has as an objective the development of an affordable computerized system to assess the gross motor skills. We try to minimize the use of wearable devices, by using the Kinect ${ }^{\mathrm{TM}}$, which allows the simultaneous measurement of the $3 \mathrm{D}$ space coordinates of the body parts and other objects such a ball. In (Tremblay et al. 2016) we reported the first prototype of the system. No information was given for the technical details of its implementation. We focused instead on the high level features and usability of the tool. A statistical analysis was performed to compare its performance to the classical human delivered tool. A new version of the application is being developed to improve the existing algorithms and develop new ones to assess all the skills of TGMD-3 (Ulrich 2013). We limit our focus on the algorithms developed to assess the horizontal jump (HJ). Similar algorithms to test the other 12 skills, not reported here, were developed. Many of these algorithms, especially those related to the ball skills (Table 1), use the color camera and a Bluetooth enabled contact sensor. Some of these skills proved to be more difficult to assess than the HJ, mostly due to occlusion, and need further refinement.

Although the accuracy of marker-less motion capture devices such as the Kinect ${ }^{\mathrm{TM}}$ is less than that of more specialized video capture systems such the $\mathrm{Vicon}^{\mathrm{TM}}$, it was shown in the literature that it is adequate for some applications involving large locomotor movements. For example it was used for motor rehabilitation (Chang et al. 2011), assessment of movement for Parkinson disease patients (Galna et al. 2014) and measurement of the knee-ankle separation ratio during the drop vertical jump (Gray et al. 2017).

\section{Materials and methods}

\section{1 System description}

The setup of the system is shown in Fig. 1. The Kinect ${ }^{\mathrm{TM}}$ is placed at a height of $0.65 \mathrm{~m}$ from the floor facing the participant such that the latter will remain in the depth of field of the system as he/she performs the movements. The origin of 
the $(\mathrm{X}, \mathrm{Y}, \mathrm{Z})$ coordinate system is located on the floor, the $\mathrm{Y}$ axis extends up and passes through the centre of the IR camera. By acquiring data from the depth camera, the Kinect ${ }^{\mathrm{TM}}$ SDK detects the user's 3D skeleton based on anatomical landmarks representing central coordinates of the body parts (Fig. 2). The software was implemented using the C\# programming language.

At the beginning of each testing session, the candidate stands at a specific location from the Kinect ${ }^{\mathrm{TM}}$, with his/her arms to his/her sides such that all the anatomical landmarks are visible on the screen. The coordinates of all corresponding body parts are recorded and stored as reference "standing position" values and are used later for different calculations in the algorithms to assess the skills. The angles of the joints, such as the knees, are also calculated by the SDK and stored.

When the test begins, the candidate performs the $\mathrm{HJ}$ and the software starts recording the $3 \mathrm{D}$ coordinates of the 25 body parts. After the execution finishes, the algorithms, explained in Section 2.2, analyse the data and the result of each performance criteria is displayed. A " 1 " is assigned for passing that criterion and a " 0 " is assigned for failure (Fig. 3).

\subsection{Algorithms development}

The TGMD-3 is a criterion-referenced test that considers the qualitative aspects of a specific skill. The assessor visually observes the movements and makes a judgment if each criterion is met or not. In order to replace the assessor based judgement by a computer based judgement, the motion is captured, digitized and algorithms are used to assess the movement based on the data describing it. For each performance criterion, an algorithm is used to determine if it satisfied or not. In order to develop the algorithms, one child and one adult performed the HJ according to the performance criteria (Table 2). Then, a recorded video of the movement and the time variations of the body parts' coordinates and joints angles were plotted to provide a better understanding of the movements involved. These graphs were used as a benchmark in developing the algorithms for assessing the HJ performed by other candidates.

The algorithms were then tested and improved by involving two children and 17 adults including students as part of the lab of an undergraduate course in human Kinetics. The kinematic curves were compared and analysed and the different parameters to be used for each criterion were estimated. The following steps are followed to develop these algorithms:

1. Based on the digitized movement, identify the HJ phases and critical points. The critical points are based on the coordinate which exhibits the most significant change and at the same time is either periodic or marks a significant phase of the skill.

2. Identify the body parts important to evaluate the HJ based on the performance criteria of TGMD and the movement execution. 
3. Establish the kinematic criteria for the body parts' coordinates and joints' angles and develop the equations based on these criteria.

4. Test the ability of the algorithms to detect a "Pass" after executing the HJ properly or to detect a "Fail" after intentionally missing a specific performance criterion. For this step the relevant coordinates and angles are plotted and quantitatively evaluated.

The procedure is iterative. After completing step 4, it might be necessary to go back to step 2, change some of the body parts used, and then go to step 3 to change the kinematic criteria.

Below, these four steps are presented in detail for developing the algorithms for the $\mathrm{HJ}$.

\subsubsection{Step1: Identification of phases and critical points}

In order to identify the HJ phases and critical points, different frames of its execution are shown in Fig. 4. This figure shows that the HJ can be divided into three distinct phases: the preparation phase (1), the aerial phase which is the actual jump (2) and the landing phase (3). It should be mentioned that in executing the HJ, the participant faces the Kinect ${ }^{\mathrm{TM}}$ and jumps towards it. The coordinate system shown in Fig. 1 is used.

The head's Y coordinate (Fig. 5), can be used to identify the different phases. During phase 1, the preparation phase, the participant is standing facing the Kinect ${ }^{\mathrm{TM}}$ and as soon as he/she receives the instruction "prepare", he/she must bend his/her knees to prepare for the jump. We define the critical point cp1 as the instant when the head's Y coordinate drops to its lowest value, which indicates that the user is about to begin the jump. In the following, the body parts' coordinates and angles are referred to by the part name, the coordinate or angle and then the time if applicable. For example, Head_ $Y_{c p l}$ refers to the Y coordinate of the head at $\mathrm{cp} 1$.

In phase 2, the user performs the jump, coming off the floor. The head's Y coordinate increases to a maximum value and then drops until the participant lands back on the floor. We define the critical point cp2 as the instant when the head's Y coordinate reaches the highest value of the whole movement. Finally, during phase 3 the participant lands on the floor and we set the critical point $\mathrm{cp} 3$ at the instant when the $\mathrm{Y}$ coordinate of the head reaches the lowest value after the maximum value occurred at cp2. The times of occurrence of the different critical points are identified, saved and used in assessing the criteria. 

mid spine and the feet. They were obtained by examining the video of performance of the HJ and the curves of the time variations of the coordinates and angles of the different body parts and relating them to the performance criteria specified in Table 2. In the following, the kinematic curves corresponding to a child successfully performing the HJ are presented to illustrate the algorithms corresponding to the different performance criteria.

\subsubsection{Step 2: Establish kinematic criteria and corresponding equations}

The first criterion analyses if the participant flexes both knees and if he/she arms are extended behind the back. We evaluate this criterion around cp1. To check if a knee is flexed or not, we compare its angle, $\alpha$, to a threshold value $\alpha_{f l e x}$ :

$$
\text { Knee_ } \alpha_{c p 1}<\alpha_{f l e x}
$$

In Fig. 6 the knees' angles are plotted versus time. At cp1 they both drop as both knees are flexed. Estimates of $\alpha_{f l e x}$ and other parameters in equations (1) to (10) are presented in Table 3. In order to determine if the arms are extended behind the back, the elbows' angles are compared to a threshold angle $\alpha_{\text {ext }}$, in addition, the $\mathrm{Z}$ coordinate of the hands should be larger than the $\mathrm{Z}$ coordinate of the shoulder by an amount $\epsilon_{Z}$ :

$$
\text { Elbow_ } \alpha_{c p 1}>\alpha_{\text {ext }}
$$

$$
\text { Hand_Z } Z_{c p 1}>\text { Shoulder_Z } Z_{c p 1}+\epsilon_{Z}
$$

In the libraries of the software, the elbow's angles are calculated from of the dot product of the elbow-hand and elbowshoulder vectors. These vectors are calculated from the values of the shoulders, elbows and hands coordinates. For the HJ, since the candidate is facing the Kinect ${ }^{\mathrm{TM}}$, in some cases the elbow or the hand, might be partly hidden from the depth camera by the shoulder. When body parts are not sufficiently visible to the depth sensor, the Kinect ${ }^{\mathrm{TM}}$ software performs interpolation to calculate their coordinates and these data points are identified as "inferred". While the inferred coordinates represent in many cases good approximations of the actual values, using them to calculate the angles might have accumulated inaccuracies and would be unreliable. In this case, either an alternative simple formula, using the visible coordinates, is used to estimate the angles, or we modify the equations to assess the criteria without relying on the angles. 
For example, as illustrated in Fig. 5 (phase 1), the child extended his arms too much to the back such that the hand and elbow are significantly hidden from the sensor by the shoulder. In Fig. 7 where we plot the $Z$ coordinates of the shoulder, elbow and hand, the values around $\mathrm{cp} 1$ are identified as inferred by the '*' markers. This is the same for the $\mathrm{X}$ and $\mathrm{Y}$ coordinates. In this case, the elbows' angles around cp1 (Fig. 10) are not reliable and cannot be used, but it is safe to assume that if the coordinates of the hand and elbow are inferred, while those of the shoulder are not, then the shoulder is hiding the elbow and hand from the depth sensor, so that the arm is extended behind the back.

To summarize, the algorithm starts by making sure the elbows and hands' joints are not inferred around the time period of interest. If this is the case, all joints are visible, and we calculate the angles and use Eqs. (2) and (3) to assess the criterion. If one of them is inferred, we use an alternative method to check the criteria using the visible joints. If both the hand and elbow joints are inferred than we assume that this criterion is satisfied.

\section{b) Criterion 2}

The second criterion analyses if the arms extend forcefully forward and upward reaching above the head. This criterion is checked during the jump, which is performed between cp1 and cp2 (phase 2). To get the forceful forward motion of the arms, the hands' $\mathrm{Z}$ coordinates have to change faster than that of the head:

$$
\left|H a n d \_Z_{c p 1}-H a n d \_Z_{c p 2}\right|>k\left|H e a d \_Z_{c p 1}-H e a d \_Z_{c p 2}\right|
$$

where $k$ is a constant, larger than 1 , indicating the forceful movement. From Fig. 8 , the change of the hands' coordinates is significantly larger than that of the head between $\mathrm{cp} 1$ and $\mathrm{cp} 2$.

Besides the forward motion (Eq. (4)) we have to assess the upward motion. For this, the hands' Y coordinates have to increase to above that of the head at cp2. The arms extension has to be forceful in order to help with the jump. To ensure this, the rate of extension has to be fast compared to the jump between $\mathrm{cp} 1$ and $\mathrm{cp} 2$. Since we used the head's Y position to characterize the jump, we compare the amount of increase of the hands' Y coordinates to that of the head between cp1 and cp2. The above conditions are expressed as:

$$
\begin{gathered}
\text { Hand_Y } Y_{c p 2}>\text { Head_Y } Y_{c p 2}+\epsilon_{Y} \\
\mid \text { Hand_Y } Y_{c p 1}-\text { Hand_Y } Y_{c p 2}|>k| \text { Head_Y } Y_{c p 1}-H e a d \_Y_{c p 2} \mid
\end{gathered}
$$

where $k>1$ indicating that the hands go up faster than the head and $\epsilon_{Y}$ is a tolerance value. In Fig. 9 it is clear that the increase of the hands' Y coordinates is larger than that of the head between $\mathrm{cp} 1$ and $\mathrm{cp} 2$, and that at $\mathrm{cp} 2$ the hands' $\mathrm{Y}$ coordinates are 
185 larger than those of the head. Finally, around $\mathrm{cp} 2$, the arms have to extend, and this can be checked by comparing the elbows' 186 angles to a threshold angle $\alpha_{e x t}$

$$
\text { Foot_Y }>\text { Foot_Y threshold }
$$

where Foot_ $Y_{\text {threshold }}=$ Foot_$_{-} Y_{\text {standing }}+\epsilon_{Y}$, and $\epsilon_{Y}$ is a tolerance value. To determine if the feet leave the floor together, the algorithm checks the first occurrence, after cp1 and before cp3, when each foot's Y coordinates are larger than the threshold value, and then checks if these occurrences happen at the same time for both feet within a tolerance value $\epsilon_{\tau}$. To determine if they land together, the algorithm checks the first occurrence, after the feet left the floor, when each foot's Y coordinate is less than the threshold value. The algorithm checks if the times of these occurrences for both feet are equal within $\epsilon_{\tau}$. Note in Fig. 11 that the first occurrence of the feet' Y coordinates above the threshold happens at close time instants and the first occurrence of the feet' Y coordinate below the threshold also happens at close time instants.

\section{d) Criterion 4}

The fourth criterion checks if both arms are forced downward during landing. To evaluate this criterion, the algorithm checks if between cp2 and cp3 the hands' Y coordinates change faster than that of the head. We also make sure that the hands' Y coordinates decrease to values lower than that of the mid-spine.

$$
\left|H a n d \_Y_{c p 2}-H a n d \_Y_{c p 3)}\right|>k\left|H e a d \_Y_{c p 2}-H e a d \_Y_{c p 3)}\right|(9)
$$




$$
\text { Hand_ } Y_{c p 3}<M i d S p i n e_{-} Y_{c p 3}-\epsilon_{Y}
$$

where $k>1$ indicating that the hands are forced downward faster than the head and $\epsilon_{Y}$ is a tolerance value. By referring to

Fig. 9, we see that the change in the hands' Y coordinates is faster that of the head and by referring to Fig. 12 we see that both

$\mathrm{Y}$ hands' coordinates decrease from $\mathrm{cp} 2$ to a value below the $\mathrm{Y}$ coordinate of the mid-spine at $\mathrm{cp} 3$. This means that the arms were forced downward during the transition from phase 2 to phase 3 .

\subsubsection{Step 4: Testing the algorithms and estimation of the parameters}

The algorithm starts by determining the relevant parameters and then proceeds to verify the corresponding equations sequentially in the order they are presented. If all equations are satisfied, a score of 1 (pass) is assigned, otherwise a score of 0 (fail) is assigned. As the algorithms were being developed, we continuously tested their ability to detect passes and fails and we modified them accordingly. Two children and 16 adults participated in testing the algorithms. Some of the adults were undergraduate students who participated in this study as part of the Lab component of a "Motor Learning" course offered in the Human Kinetics Program.

The "standing position" coordinates, along with other data entered about the user such as height and age, can be used to estimate some reasonable values of the different tolerances and thresholds used in the algorithms.

Table 3 gives the values of the parameters in equations (1) to (10) and the way they were evaluated. For example, for equations (1),(2) and (7) the flexion or extension of the joint is assessed by comparing its angle to a threshold value defined from the standing position by a reasonable angle for flexion or extension. For equations (4),(6) and (9) the forceful movement is assessed by setting a minimum comparison ratio of $k=2$. The tolerance values used in equations (3),(5),(8) and (10) were also reasonable values and similarly to the previous parameters, they worked for all participants in this investigation. They can be changed, if needed, for example when testing with a much younger group or a more conservative assessment is wanted.

\section{Results and discussion}

To test the algorithms, the HJ was performed multiple times properly in order to verify whether a "pass" would be returned for each criterion. We then performed each criterion of the HJ incorrectly, in order to verify whether a "fail" would be assigned for each. For each test, the data containing the time variation of the coordinates of each joint is stored. The kinematic curves are plotted and examined by checking how the algorithms described by equations (1) to (10) apply. The performance of the HJ was also videotaped and examined along with the curves. 

HJ compared to human assessment. This study confirms what has been reported in the literature, of the potential of markerless, low cost motion capture systems, such as the Kinect ${ }^{\mathrm{TM}}$, to study large locomotor movements (Chang et al. 2011; Galna et al. 2014; Gray et al. 2017). One possible reported limitation of the Kinect ${ }^{\mathrm{TM}}$ is its frame rate of $30 \mathrm{fps}$, corresponding to a sampling time of $T=1 / 30 \mathrm{~s}$, which might be insufficient to resolve fast movement events. For example its performance was compared to that of a Vicon ${ }^{\mathrm{TM}}$ system for measurement of the knee-ankle separation ratio for a vertical jump during initial contact and peak flexion (Gray et al. 2017). The authors concluded that even though the events considered were fast, the Kinect ${ }^{\mathrm{TM}}$ was able to accurately measure this parameter when the right frames are used. For the present study, by looking at the most time sensitive events, we see for example that the forceful movement of the arms duration is about 12 times the sampling time (Fig. 8). Also, from Figure 11, for the third performance criterion that "Both feet come off the floor together and land together", we set a tolerance $\epsilon_{\tau} \simeq 0.1 \mathrm{~s}$ (Table 3), which is 3 times the sampling time.

\section{Conclusions and future work}

A computer based application using a motion capture system and Bluetooth enabled contact sensors to replace the human assessment of the Test of Gross Motor Development, is being developed. We presented a set of algorithms based on is needed. the expected kinematic behavior of the relevant body parts while performing the HJ. These algorithms were successful in assessing the different performance criteria. It is recognized that the tests performed are used only to evaluate the algorithms and are not intended to validate the computerized application as a testing tool.

Development of the algorithms for the other 12 skills is in progress and more testing of these algorithms and refinement

\section{References}

263 Bisi, M. C., Panebianco, G. P., Polman, R., and Stagni, R. 2017. Objective assessment of movement competence in children using wearable sensors: An instrumented version of the TGMD-2 locomotor subtest. Gait \& Posture, 56: 
Bonifacci, P. 2004. Children with low motor ability have lower visual-motor integration ability but unaffected perceptual skills. Human Movement Science, 23(2): 157-168. doi:10.1016/j.humov.2004.08.002

Booth, M. L. et al. 1999. Mastery of fundamental motor skills among New South Wales school students: prevalence and sociodemographic distribution. Journal of Science and Medicine in Sport, 2(2): 93-105. doi:10.1016/s1440-2440(99)80189-3

Chang, Y., Chen, S., and Huang, J. D. 2011. A Kinect-based system for physical rehabilitation: A pilot study for young adults with motor disabilities. Research in Developmental Disabilities, 32(6): 2566-2570.

\section{doi:10.1016/j.ridd.2011.07.002}

Frey, G. C., and Chow, B. 2006. Relationship between BMI, physical fitness, and motor skills in youth with mild intellectual disabilities. International Journal of Obesity, 30: 861-867. doi:10.1038/sj.ijo.0803196

Galna, B. et al., 2014. Accuracy of the Microsoft Kinect sensor for measuring movement in people with Parkinson's disease. Gait \& Posture, 39(4): 1062-1068. doi:10.1016/j.gaitpost.2014.01.008

Goodway, J. D., Crowe, H., and Ward, P. 2003. Effects of motor skill instruction on fundamental motor skill development. Adapted Physical Activity Quarterly, 20(3): 298-314. doi:10.1123/apaq.20.3.298

Gray, A. D. et al. 2017. Development and validation of a portable and inexpensive tool to measure the drop vertical jump using the Microsoft Kinect V2. Sports Health , 9(6): 537-544. doi:10.1177/1941738117726323

Griffiths, A., Toovey, R., Morgan, P. E., and Spittle, A. J. 2018. Psychometric properties of gross motor assessment tools for children: a systematic review. BMJ Open, 8(10): e021734. doi:10.1136/bmjopen-2018021734

Grimpampi, E., Masci, I., Pesce, C., and Vannozzi, G. 2016. Quantitative assessment of developmental levels in overarm throwing using wearable inertial sensing technology. Journal of Sports Sciences, 34(18): 17591765. doi:10.1080/02640414.2015.1137341

Masci, I., Vannozzi, G., Getchell, N., and Cappozzo, A. 2012. Assessing hopping developmental level in childhood using wearable inertial sensor devices. Motor Control, 16(3): 317-328. doi:10.1123/mcj.16.3.317

McCormick, A. et al. 2007. Stability of the Gross Motor Function Classification System in Adults with Cerebral Palsy. Developmental Medicine \& Child Neurology, 49(4): 265-269. doi:10.1111/j.1469-8749.2007.00265.x

Skinner, R.A., and Piek, J.P. 2001. Psychosocial implications of poor motor coordination in children and adolescents. Human Movement Science, 20(1-2): 73-94. doi:10.1016/s0167-9457(01)00029-X

Tremblay, L. et al. 2016. Description and Features of a New Computerized Gross Motor Skills Measurement. Journal of Technologies and Human Usability, 12(3-4): 31-49. doi:10.18848/2381-9227/cgp/v12i3-4/31-49 Ulrich, D. 2013. The Test of Gross Motor Development - 3 (TGMD-3): Administration, scoring, and international norms. Spor Bilimleri Dergisi, 24(2): 27-33. 
299 Voelcker-Rehage, C., 2008. Motor-skill learning in older adults-a review of studies on age-related differences. 300 European Review of Aging and Physical Activity , 5(1): 5-16. doi:10.1007/s11556-008-0030-9

301 Zecevic, C. A., Tremblay, L., Lovsin, T. and Michel, L. 2010. Parental influence on young children's physical 302 activity. International Journal of Pediatrics. doi:10.1155/2010/468526 


\section{$304 \quad$ Figures captions}

305 Figure 1. Physical setup of system.

306 Figure 2. Anatomical Landmarks tracked by Kinect ${ }^{\mathrm{TM}}$.

307 Figure 3. Examples of a horizontal jump test results.

308 Figure 4. Horizontal jump movement.

309 Figure 5. Y coordinate of head and critical points: cp1, cp2 and cp3.

$310 \quad$ Figure 6. Angles of knees.

311 Figure 7. Z coordinates of left hand, elbow and shoulder.

312 Figure 8. Z coordinates of head and hands.

313 Figure 9. Y coordinates of head and hands.

314 Figure 10. Angles of elbows.

315 Figure 11. Y coordinates of feet.

316 Figure 12 . Y coordinates of hands and mid-spine. 
317 Table 1. Skills of the Test of Gross Motor Development (TGMD-3) (Ulrich 2013).

\begin{tabular}{ll}
\hline $\begin{array}{l}\text { Locomotor } \\
\text { skills }\end{array}$ & Ball kills \\
\hline Run & $\begin{array}{l}\text { Two-hand strike of a stationary } \\
\text { ball }\end{array}$ \\
\hline Gallop & $\begin{array}{l}\text { One-hand strike of self-bounced } \\
\text { ball }\end{array}$ \\
\hline Hop & One-hand stationary dribble \\
\hline Skip & Two-hand catch \\
\hline $\begin{array}{l}\text { Horizontal } \\
\text { Jump }\end{array}$ & Kick a stationary ball \\
\hline Slide & Overhand throw \\
\hline & Underhand throw \\
\hline
\end{tabular}

Table 2. Performance criteria for the horizontal jump (Ulrich 2013).

1. Prior to take off both knees are flexed and arms are extended behind the back.

2. Arms extend forcefully forward and upward reaching above the head.

3. Both feet come off the floor together and land together.

4. Both arms are forced downward during landing. 
Table 3. Determination of parameters for Eqs. (1) to (10).

\begin{tabular}{|c|c|}
\hline $\begin{array}{l}\text { Equation \& } \\
\text { parameter }\end{array}$ & Determination of parameters \\
\hline $\begin{array}{ll}\text { Eq. } & (1) \\
& \alpha_{f l e x}\end{array}$ & $\begin{array}{l}\text { A minimum noticeable flexing angle of the } \\
\text { knee from the standing position is around } \\
20^{\circ} \\
\text { We set } \alpha_{\text {flex }}=\alpha_{\text {knee standing }}-20^{\circ} \text {. }\end{array}$ \\
\hline $\begin{aligned} \text { Eq. } & (2) \\
& \alpha_{e x t}\end{aligned}$ & $\begin{array}{l}\text { An extended arm should have an angle } \\
\text { close to the standing position value. We } \\
\text { allow for a tolerance of } 10^{\circ} \text { for any } \\
\text { participant. We set } \alpha_{\text {ext }}=\alpha_{\text {elbow_standing }}-10^{\circ} \text {. }\end{array}$ \\
\hline $\begin{array}{r}\text { Eq. (3) } \\
\qquad \epsilon_{Z}\end{array}$ & $\begin{array}{l}\text { Geometrical estimate gives a minimum } \\
\text { value of } \epsilon_{Z} \text { to be around } 60 \% \text { of the } \\
\text { distance between the hand and the } \\
\text { shoulder in the standing position for any } \\
\text { participant. }\end{array}$ \\
\hline $\begin{array}{c}\text { Eq. (4) } \\
k\end{array}$ & $\begin{array}{l}\text { We set a minimum value, } k \approx 2 \text {, for forceful } \\
\text { movement. }\end{array}$ \\
\hline Eq. (5) $\epsilon_{Y}$ & $\begin{array}{l}\text { The average height of human head is } \sim 24 \\
\mathrm{~cm} \text {, and the head's coordinate is measured } \\
\text { at the middle of the head. Setting a } \\
\text { tolerance value of } 5 \mathrm{~cm}: \epsilon_{Y} \approx 17 \mathrm{~cm} .\end{array}$ \\
\hline $\begin{array}{c}\text { Eq. (6) } \\
k\end{array}$ & Similar to Eq. (4): $k \approx 2$ \\
\hline $\begin{aligned} & \text { Eq. }(7) \\
& \alpha_{e x t} \\
&\end{aligned}$ & Similar to Eq. (2) $\alpha_{\text {ext }}=\alpha_{\text {elbow_standing }}-10$ \\
\hline $\begin{array}{l}\text { Eq. }(8) \\
\text { Foot } Y_{\text {standing }}\end{array}$ & $\begin{array}{l}\text { Foot_Y } Y_{\text {standing is determined from the }} \\
\text { standing position by taking an average } \\
\text { value. }\end{array}$ \\
\hline$\epsilon_{\mathrm{Y}}$ & $\begin{array}{l}\text { A value } \epsilon_{\mathrm{Y}}=8 \mathrm{~cm} \text { is enough for all } \\
\text { participants to show that the feet left the } \\
\text { floor. }\end{array}$ \\
\hline$\epsilon_{\tau}$ & $\begin{array}{l}\text { A value } \epsilon_{\tau} \simeq 0.1 \mathrm{~s} \text { would suggest the feet } \\
\text { left the floor or landed together. }\end{array}$ \\
\hline $\begin{array}{c}\text { Eq. (9) } \\
k\end{array}$ & Similar to Eq. (4) $: k \approx 2$ \\
\hline $\begin{array}{r}\text { Eq. (10) } \\
\epsilon_{Y}\end{array}$ & $\begin{array}{l}\text { A value } \epsilon_{Y}=10 \mathrm{~cm} \text { clearly shows that } \\
\text { the arms are down for any participant. }\end{array}$ \\
\hline
\end{tabular}




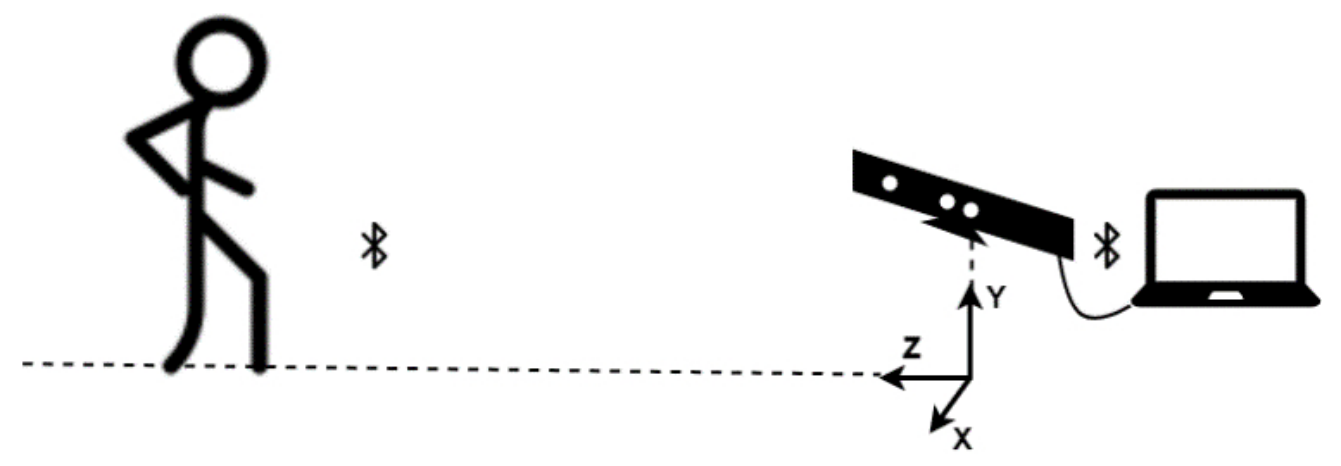

Figure 1. Physical setup of system.

$87 \times 31 \mathrm{~mm}(220 \times 220$ DPI $)$ 


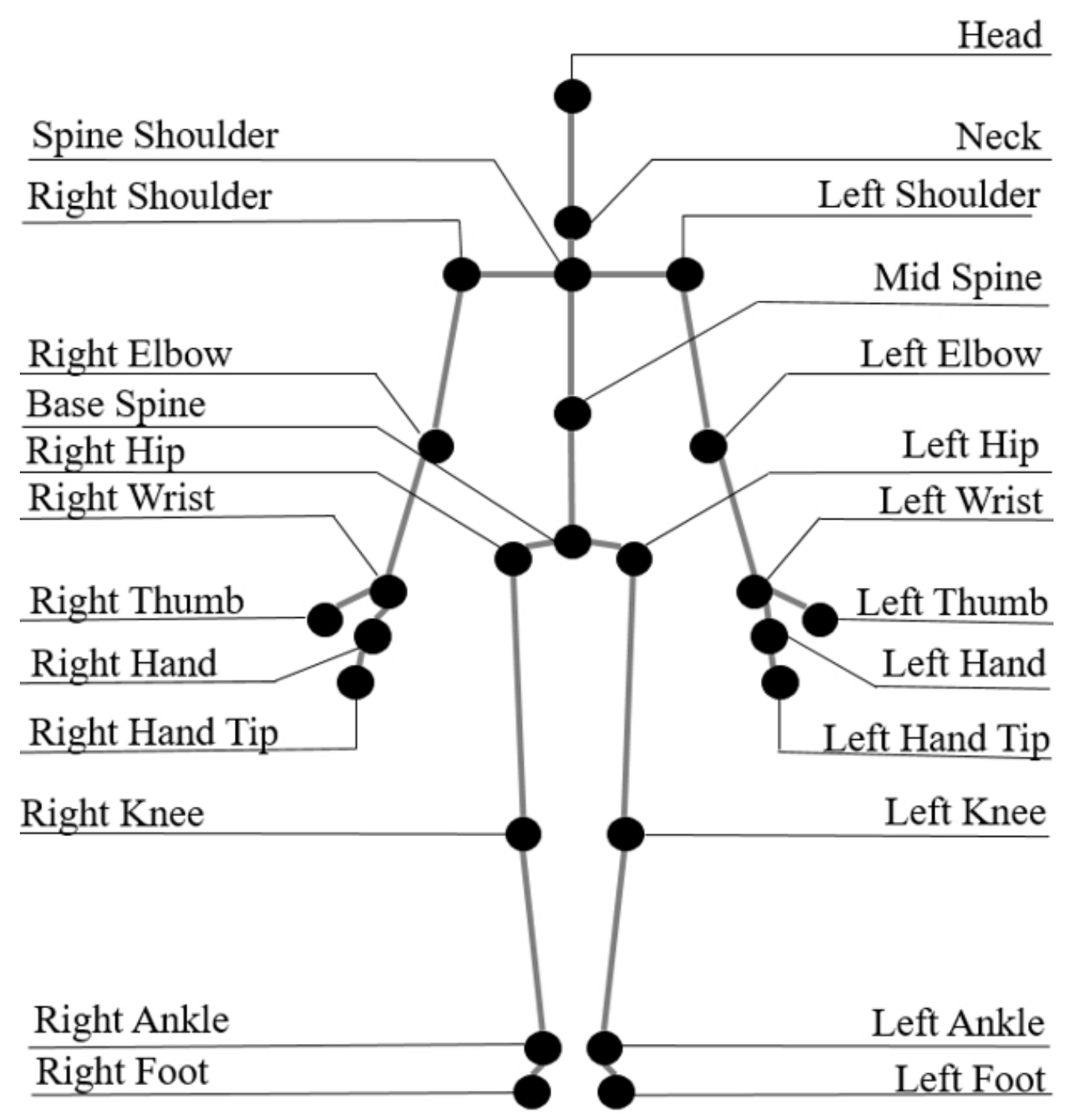

Figure 2. Anatomical Landmarks tracked by KinectTM.

$104 \times 113 \mathrm{~mm}(150 \times 150 \mathrm{DPI})$ 


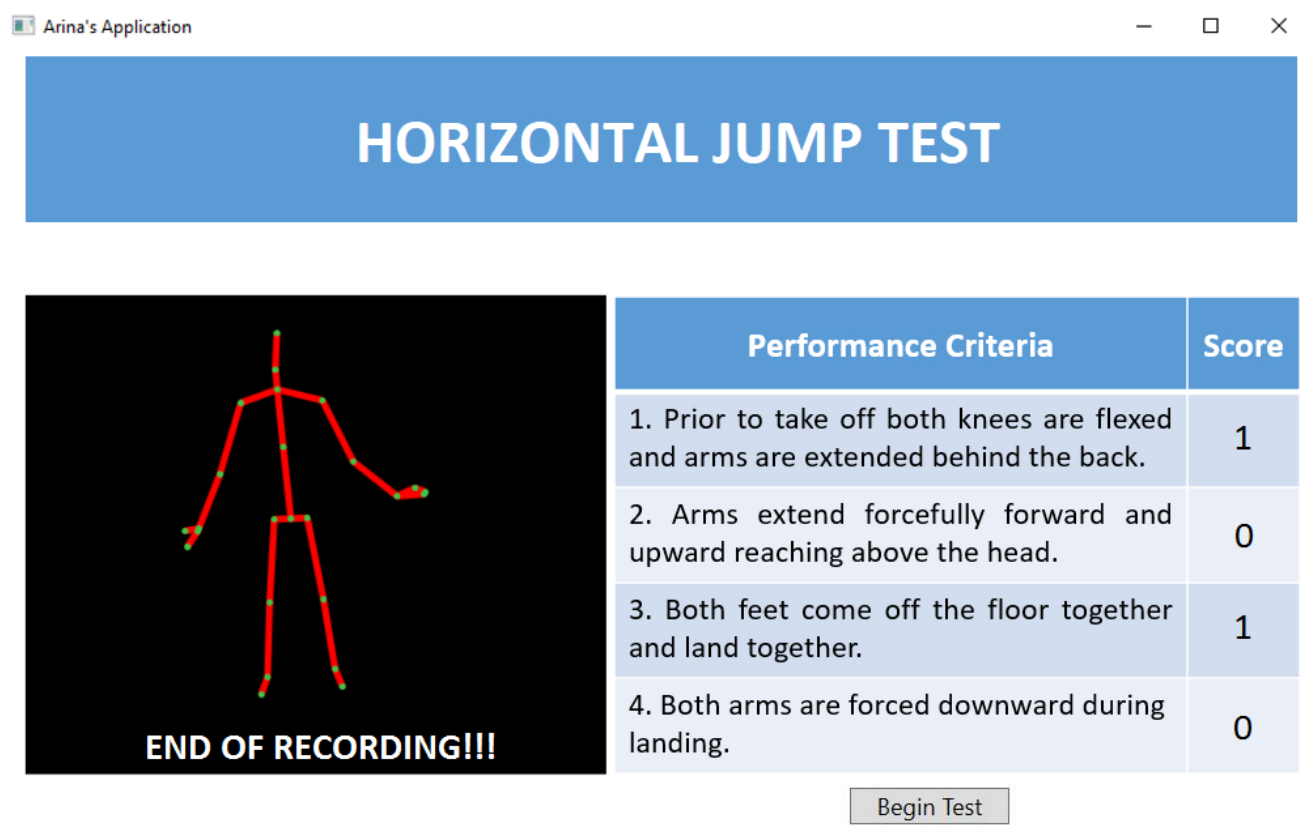

Figure 3. Examples of a horizontal jump test results.

$263 \times 168 \mathrm{~mm}(96 \times 96 \mathrm{DPI})$ 


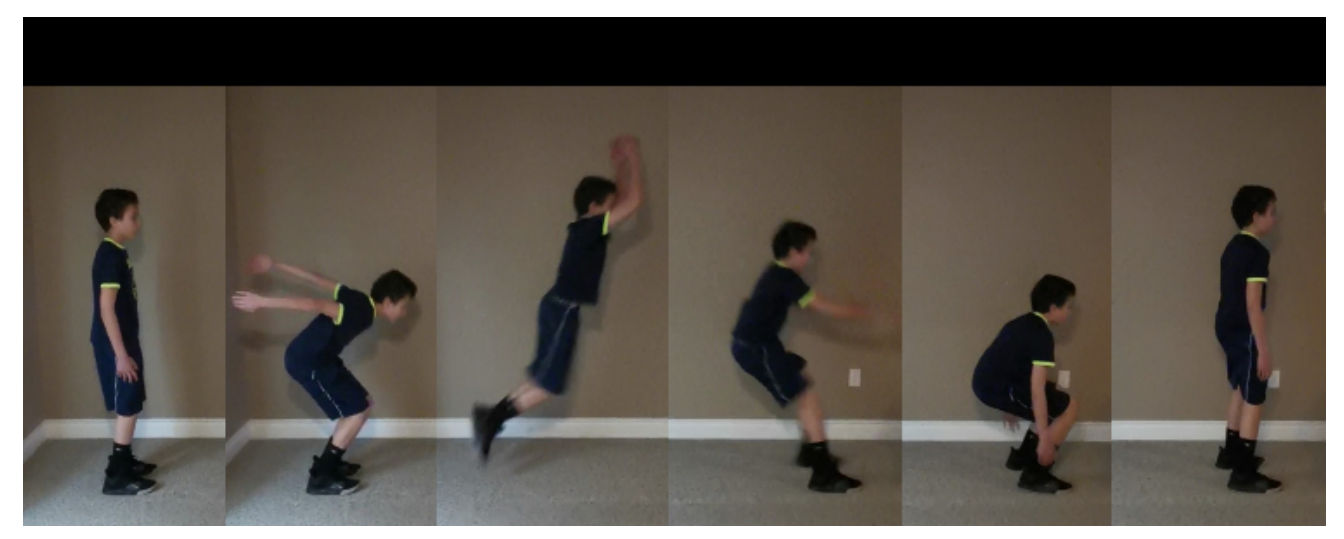

Figure 4. Horizontal jump movement.

$86 \times 34 \mathrm{~mm}(220 \times 220$ DPI $)$ 


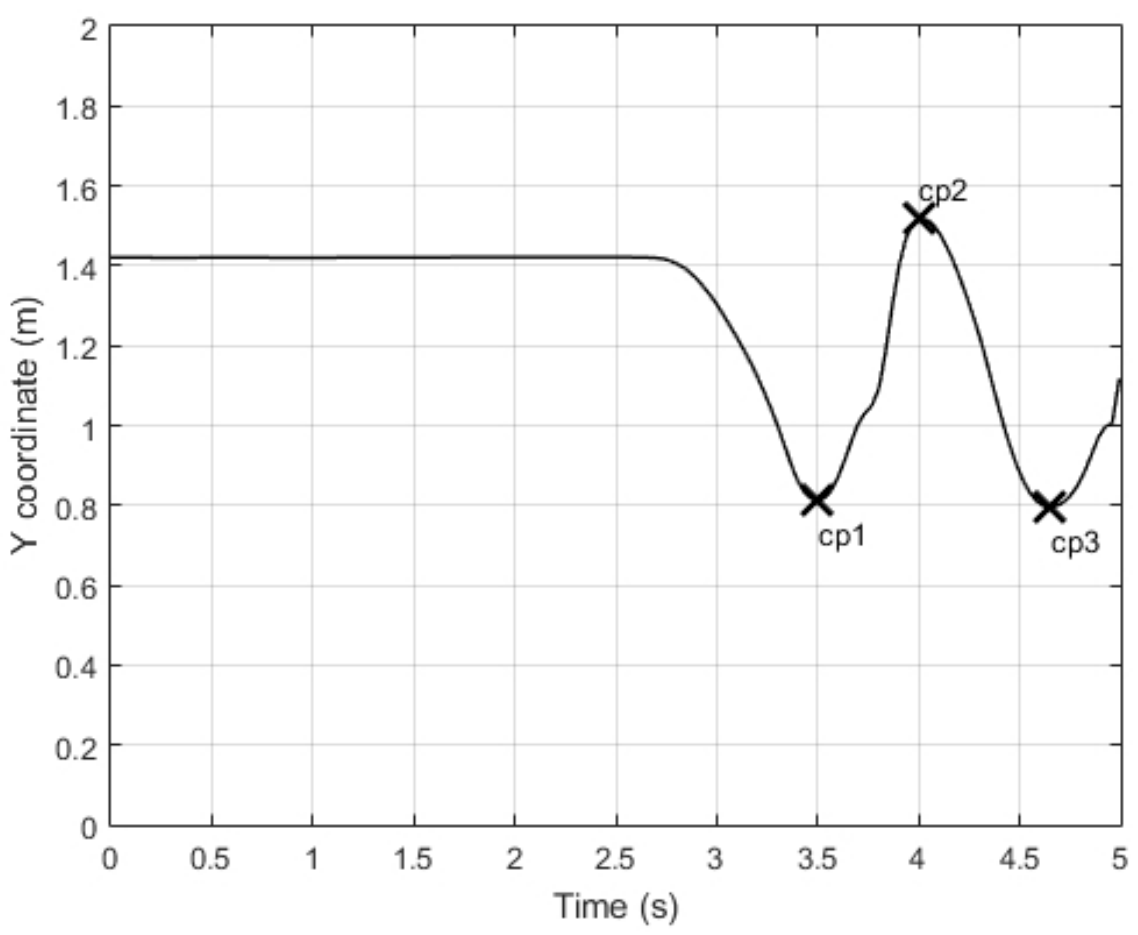

Figure 5. Y coordinate of head and critical points: $\mathrm{cp} 1, \mathrm{cp} 2$ and $\mathrm{cp} 3$. $148 \times 111 \mathrm{~mm}(96 \times 96 \mathrm{DPI})$ 


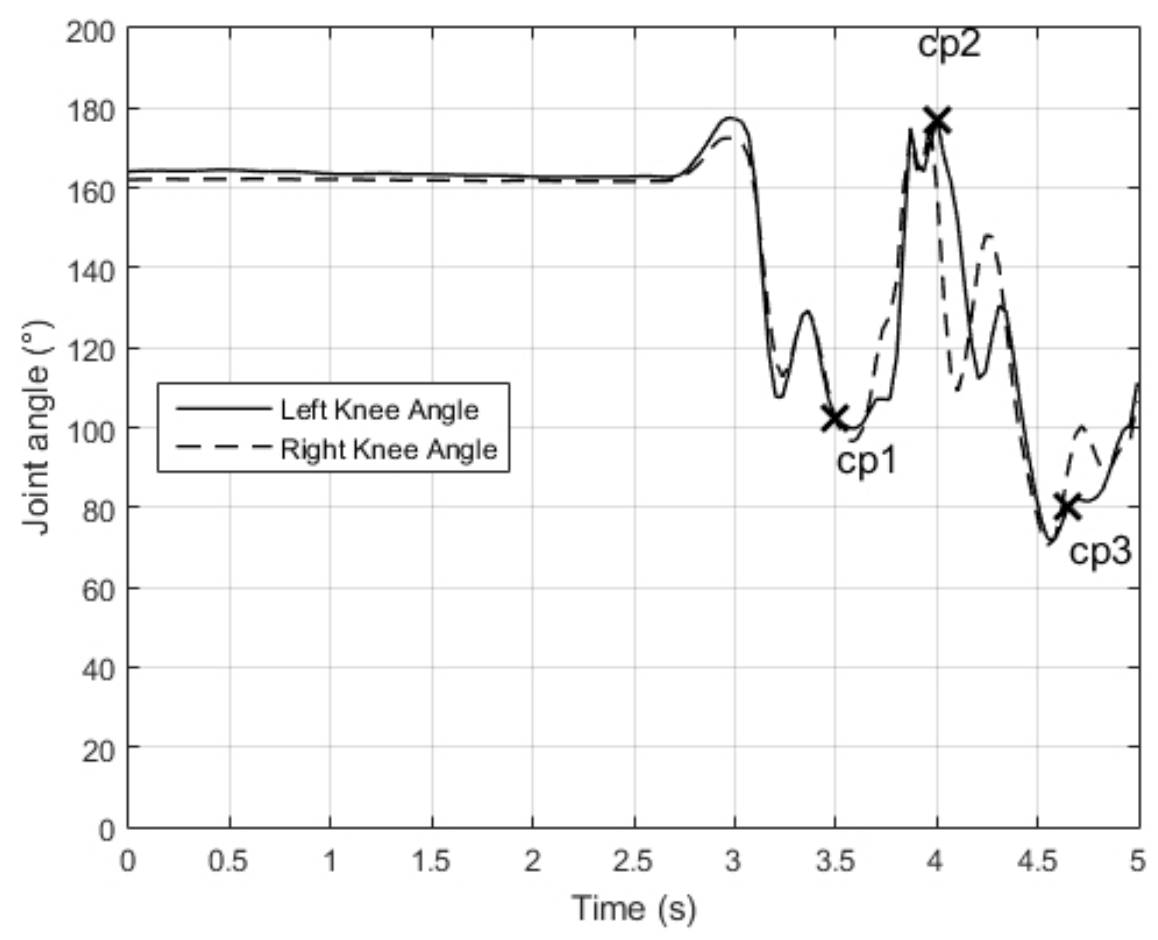

Figure 6. Angles of knees.

$148 \times 111 \mathrm{~mm}(96 \times 96 \mathrm{DPI})$ 


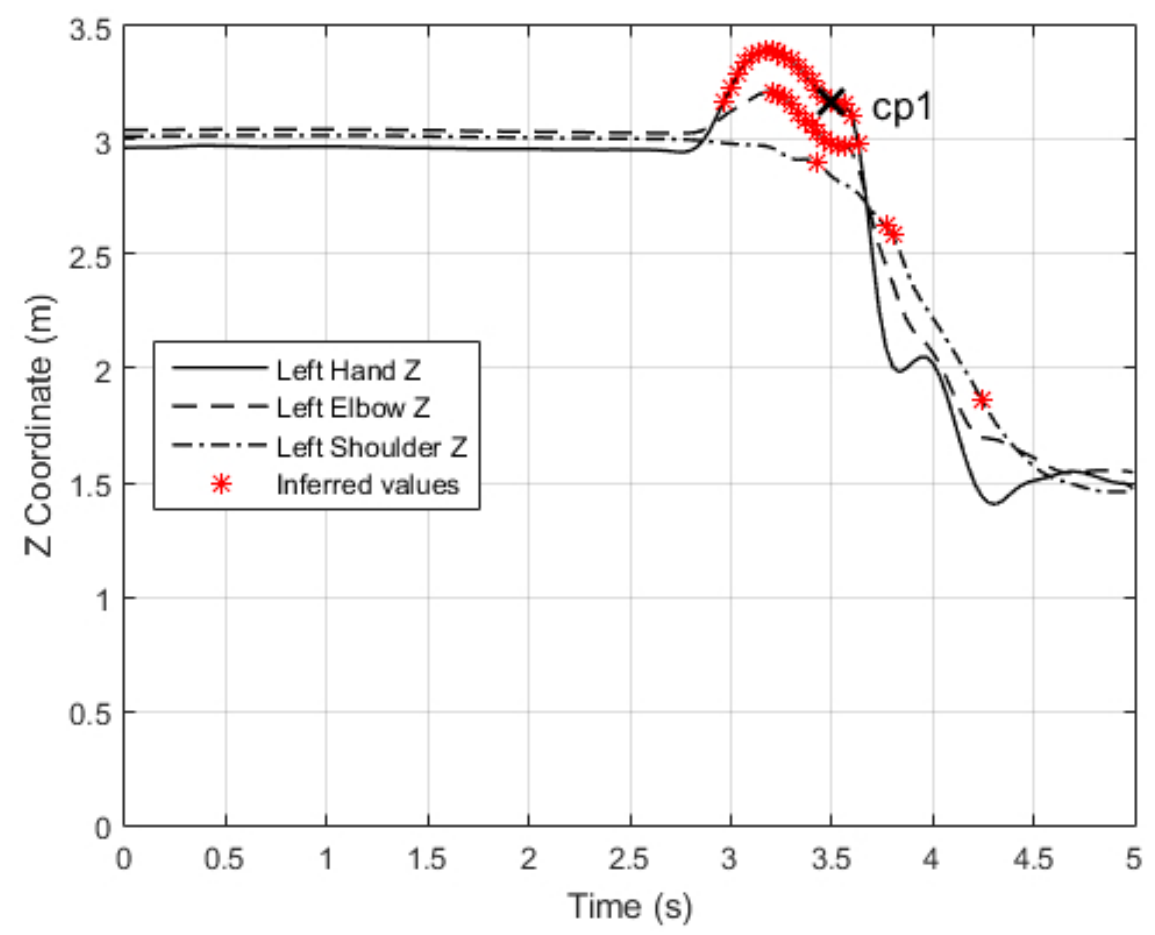

Figure 7. Z coordinates of left hand, elbow and shoulder.

$148 \times 111 \mathrm{~mm}(96 \times 96 \mathrm{DPI})$ 


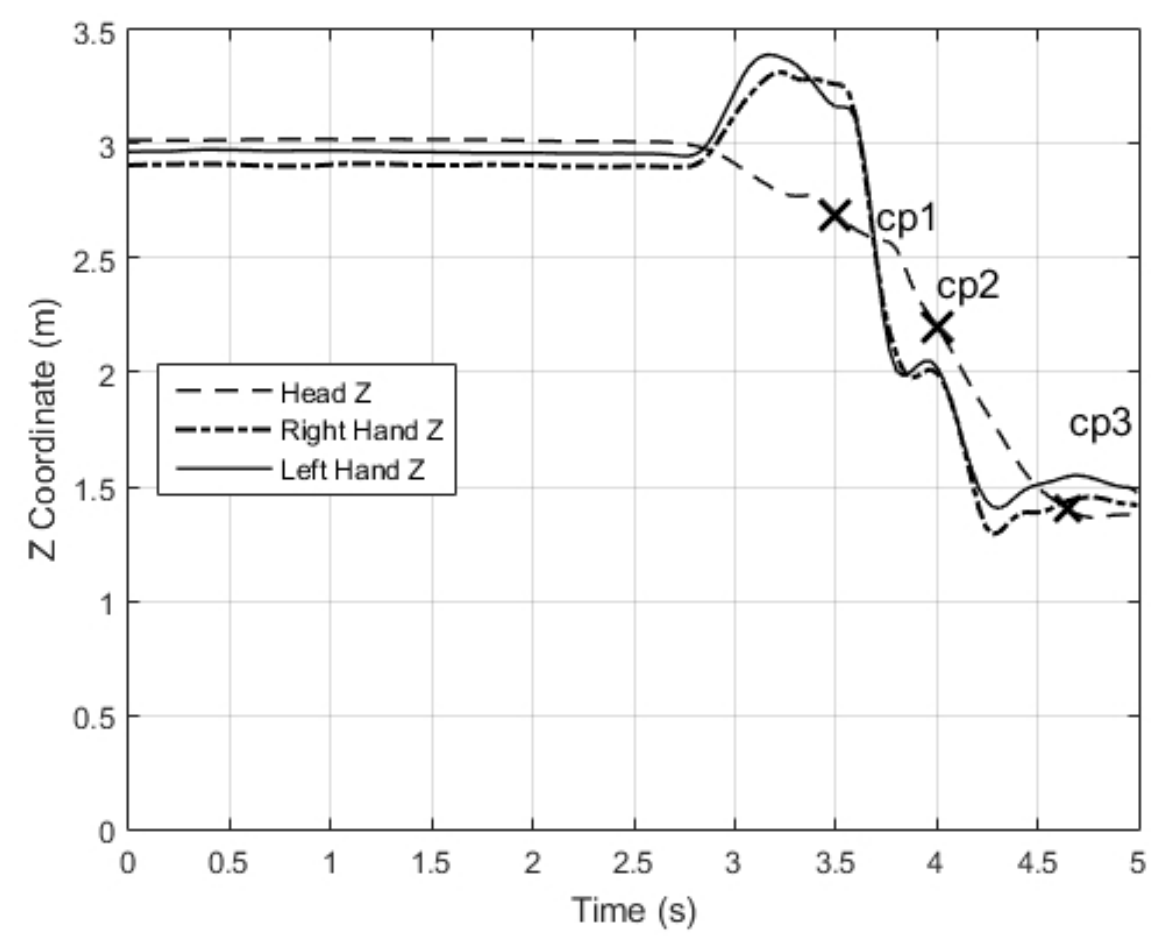

Figure 8. Z coordinates of head and hands.

$148 \times 111 \mathrm{~mm}(96 \times 96 \mathrm{DPI})$ 


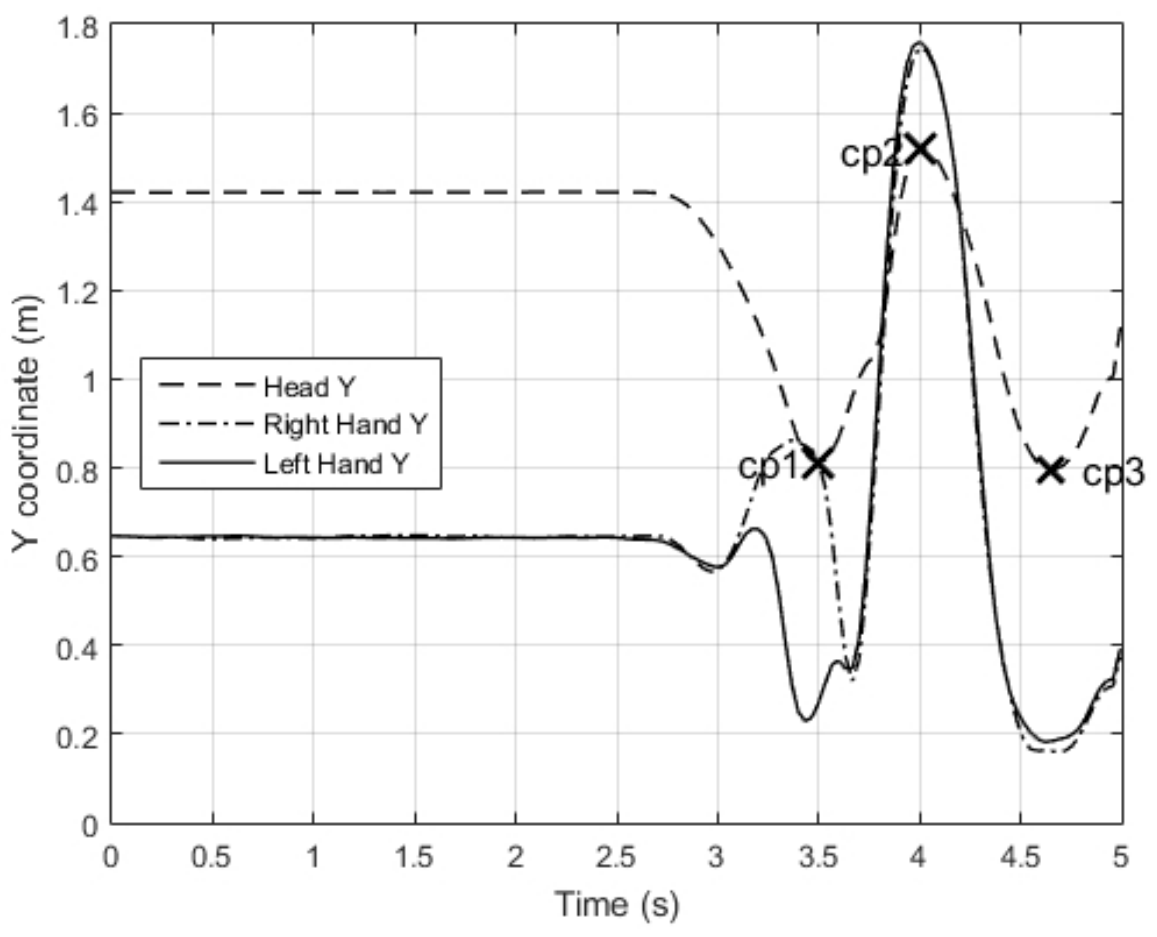

Figure 9. Y coordinates of head and hands.

$148 \times 111 \mathrm{~mm}(96 \times 96 \mathrm{DPI})$ 


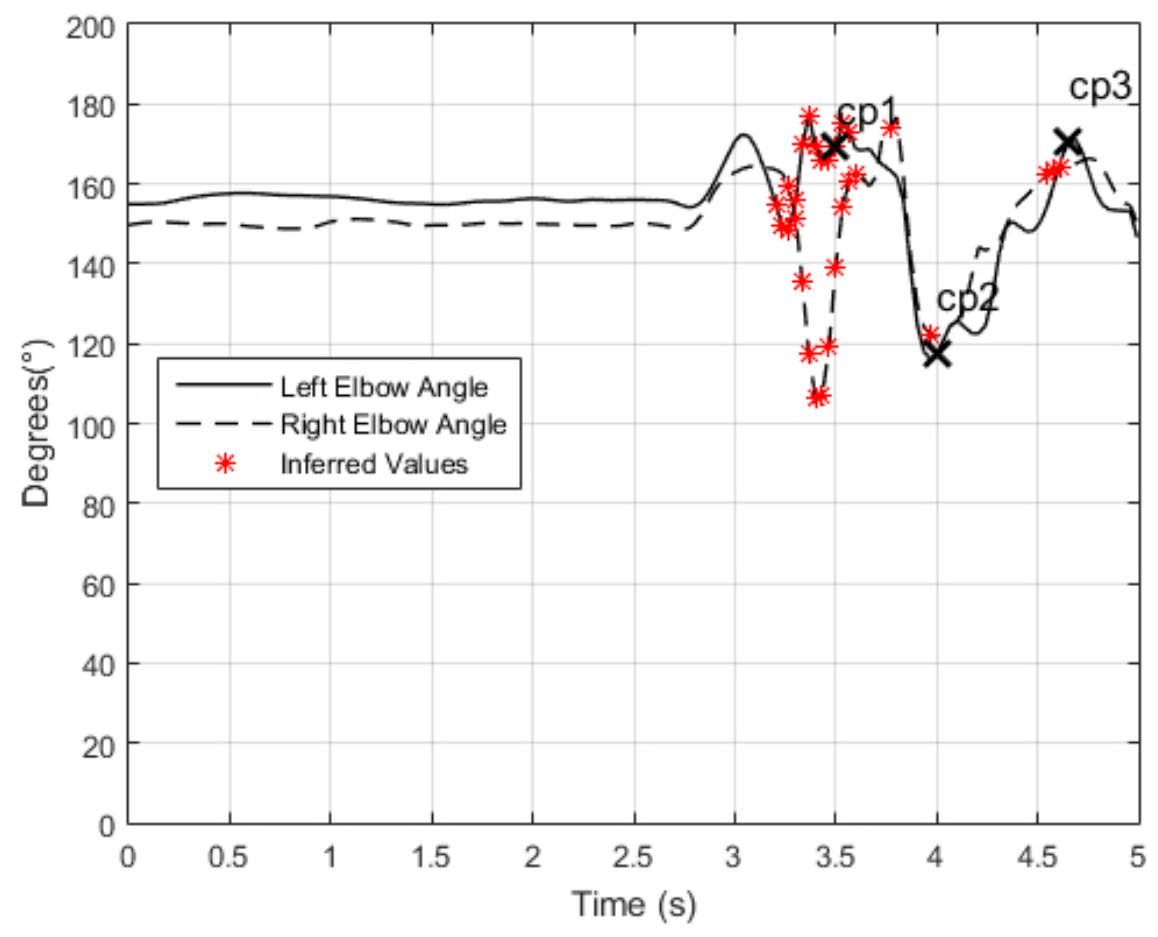

Figure 10. Angles of elbows.

$148 \times 111 \mathrm{~mm}(96 \times 96 \mathrm{DPI})$ 


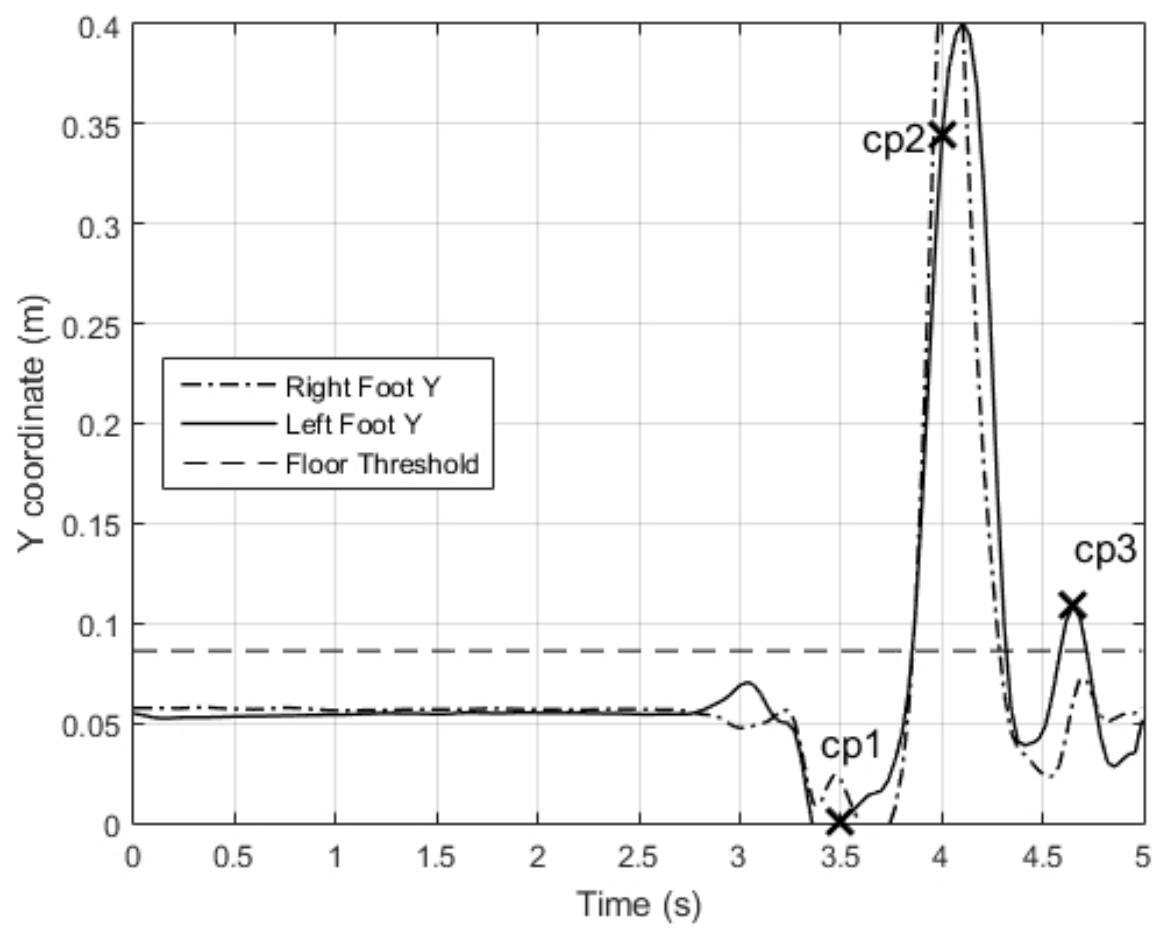

Figure 11. Y coordinates of feet.

$148 \times 111 \mathrm{~mm}(96 \times 96 \mathrm{DPI})$ 


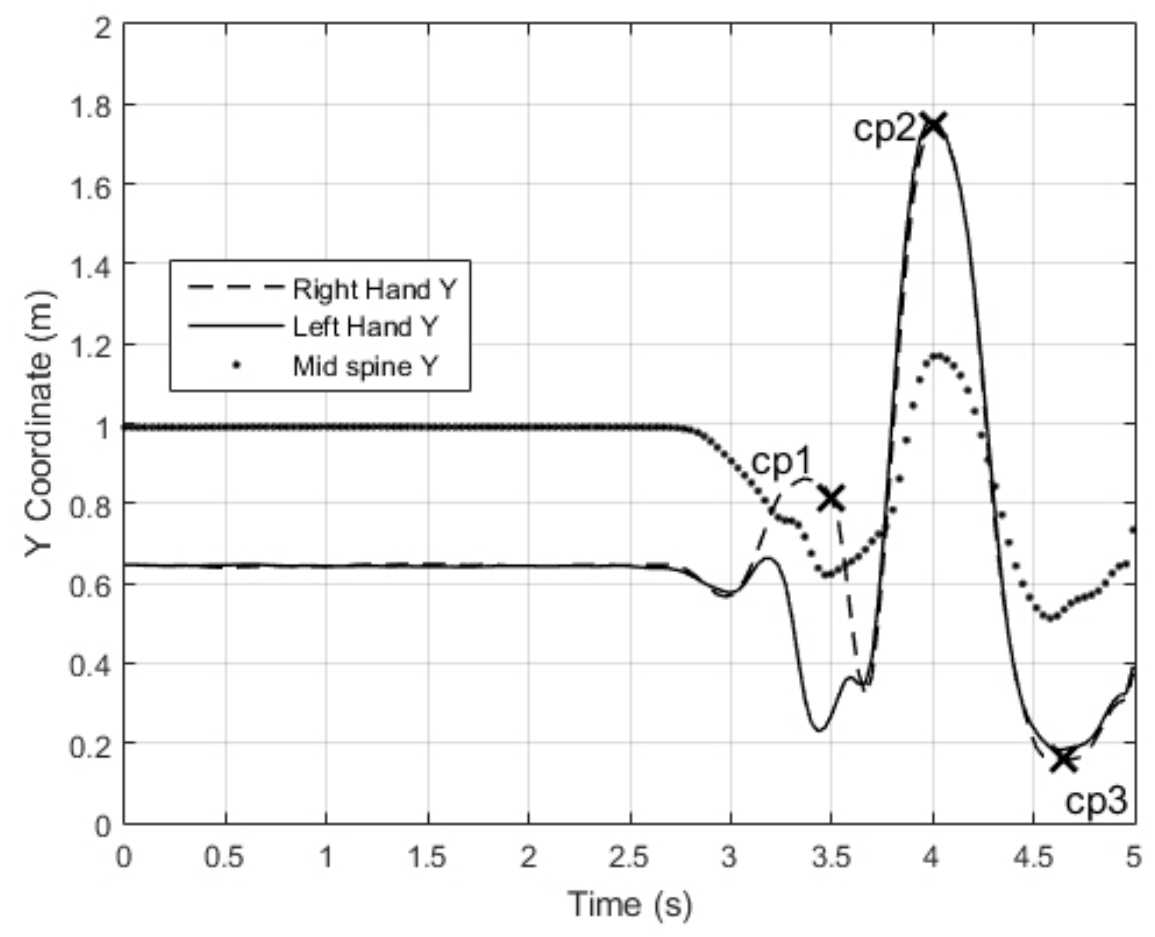

Figure 12. Y coordinates of hands and mid-spine.

$148 \times 111 \mathrm{~mm}(96 \times 96 \mathrm{DPI})$ 